IRA-International Journal of Applied Sciences ISSN 2455-4499; Vol.07, Issue 02 (2017)

Institute of Research Advances

Pg. no. 75-94

http://research-advances.org/index.php/IRAJAS

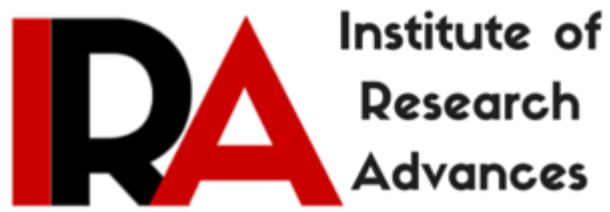

\title{
Evaluation of Craniofacial Morphology in Subjects with Down's Syndrome: A Cephalometric Study
}

Dr. Pradeep Subbaiah

Assistant Professor, Department of Orthodontics

JSS Dental College and Hospital, Mysore, Karnataka, India.

Type of Review: Peer Reviewed.

DOI: http://dx.doi.org/10.21013/jas.v7.n2.p4

\section{How to cite this paper:}

Subbaiah, P. (2017). Evaluation of Craniofacial Morphology in Subjects with Down's Syndrome: A Cephalometric Study. IRA-International Journal of Applied Sciences (ISSN 2455-4499), 7(2), 75-94. doi:http://dx.doi.org/10.21013/jas.v7.n2.p4

(C) Institute of Research Advances

\section{(cc) BY-NG}

This work is licensed under a Creative Commons Attribution-Non Commercial 4.0 International License subject to proper citation to the publication source of the work.

Disclaimer: The scholarly papers as reviewed and published by the Institute of Research Advances (IRA) are the views and opinions of their respective authors and are not the views or opinions of the IRA. The IRA disclaims of any harm or loss caused due to the published content to any party. 
Down's syndrome is a disease entity in which the defect in mental development is associated in a unique way with a large number of physical defects. ${ }^{1,2}$ It is a condition resulting from an extra chromosome 21 and thus the condition is also known as Trisomy 21. It is considered one of the most frequent genetic causes of mental retardation. ${ }^{3}$

The incidence of Down's syndrome is estimated to be between 1.6 to 2.5 per 1000 live births and increases with increase in maternal age. ${ }^{4,59,10}$ Mongoloids usually exhibit retarded motor and language skills, with an IQ range from extremely low to $45-55$. 6,711,12

Cephalometry has now been firmly established as the most essential procedure for gaining knowledge of the complexities of dentofacial skeletal pattern. By utilizing cephalometric radiography, whereby description, morphologic outline, measurement and change in growth of the skull can be fairly accurately ascertained, it might be possible to observe some of the subtle

The present study is an attempt to determine cephalometrically whether the group of individuals with Down's syndrome have a distinctive craniofacial phenotype. The growth of the craniofacial area in the presence of the extra chromosome is also examined.

\section{AIMS AND OBJECTIVES}

- The aim of this study is to analyze cephalometrically the morphology of cranial base, midface and dental characteristics in subjects with Down's syndrome and compare the same with that of normal.

- To evaluate the growth of the cranial base and maxilla in subjects with Down's syndrome and to compare the same with that of normal.

\section{Material and methods-}

For the present study a sample of 32 (16 males and 16 females) patients with Down's syndrome ranging in age from 6 to 18 years were selected. The sample was further broken down into the following age groups: 6 to 12 years and 12 to 18 years. All the patients were verified trisomic Mongoloid by virtue of karyotype examination. ${ }^{4,7}$ The consent form for the study was signed by the parents.

The control group consisted of 32 normal individuals (16 males and 16 females) and was balanced with the trisomy 21 group as far as possible for age and sex.

All the members of the control group had Class I or end to end molar relationships. The study was approved by the ethics committee of J.S.S. Dental College and Hospital. 
The cephalometric analysis comprised of the following variables:

A) Skeletal measurements

1) $\mathrm{NSBa}$

Male

2) $\mathrm{S}-\mathrm{N}$

$130^{\circ}+6$

3) $\mathrm{S}-\mathrm{Ba}$

$83 \mathrm{~mm}+4$

$50 \mathrm{~mm}+4$

4) $\mathrm{Ba}-\mathrm{N}$

$120 \mathrm{~mm} \pm 4$

$82^{0}+4$

5) $\mathrm{SNA}$

$5^{0}+2$

Female

6) $\mathrm{Pal} \mathrm{Pl}-\mathrm{NSe}$

$60 \mathrm{~mm}+4$

$130^{\circ}+6$

7) $\mathrm{N}$ - ANS

$62 \mathrm{~mm}+4$

$77 \mathrm{~mm}+4$

$46 \mathrm{~mm}+4$

$112 \mathrm{~mm}+5$

$82^{0}+4$

$5^{0}+2$

8) $\mathrm{PNS}$ - ANS

$56 \mathrm{~mm}+3$

$57 \mathrm{~mm}+4$

B) Dental Measurements

1) $\mathrm{U} 1-\mathrm{NA}\left({ }^{0}\right)$

2) $\mathrm{U} 1-\mathrm{NA}(\mathrm{mm})$

3) Pal pl-U1

4) $\mathrm{Pal} \mathrm{pl-U6}$

5) U1-SN

6) U1-Palpl
$22^{0}+6$

$4 \mathrm{~mm}+3$

$33 \mathrm{~mm}+3$

$28 \mathrm{~mm}+3$

$104^{0}+6$

$112^{0} \pm 6$
$22^{0}+6$

$4 \mathrm{~mm}+3$

$33 \mathrm{~mm}+3$

$25 \mathrm{~mm} \pm 2$

$104^{0}+6$

$112^{\overline{0}} \pm 6$

Table: 1Distribution of the subjects by Age, Sex and Group

\begin{tabular}{|l|l|l|l|l|}
\hline \multirow{2}{*}{ Age } & Sex & \multicolumn{2}{|l|}{ Group } & \multirow{2}{*}{ Total } \\
\cline { 3 - 5 } & & $\begin{array}{l}\text { Down's Syndrome } \\
\text { (DS) }\end{array}$ & Normal (N) & \\
\hline \multirow{2}{*}{ 6-12 years } & Male (M) & 8 & 8 & 16 \\
\cline { 2 - 5 } & Female (F) & 8 & 8 & 16 \\
\hline 12-18 years & Male (M) & 8 & 8 & 16 \\
\cline { 2 - 5 } & Female (F) & 8 & 8 & 16 \\
\hline Total (T) & & 16 & 16 & 32 \\
\hline
\end{tabular}


Table 2: Mean NSBa values of male and female subjects belonging to different age group in Down's syndrome and Normal groups along with the results of $F$ test

\begin{tabular}{|l|l|l|l|l|l|l|l|}
\hline \multirow{2}{*}{$\begin{array}{l}\text { Age } \\
\text { groups }\end{array}$} & \multicolumn{2}{l}{ Sex } & \multicolumn{2}{l}{ DS } & \multicolumn{2}{l|}{ Normal } & \multicolumn{2}{l|}{ Overall } \\
\cline { 1 - 6 } 6-12 yrs & & Mean & S.D & Mean & S.D & Mean & S.D \\
\cline { 2 - 8 } & M & 133.5000 & 2.9277 & 126.1250 & 4.7940 & 129.8125 & 5.4064 \\
\cline { 2 - 8 } & F & 140.0000 & 2.6186 & 128.3750 & 3.3354 & 134.1875 & 6.6655 \\
\cline { 2 - 8 } & T & 136.7500 & 4.2973 & 127.2500 & 4.1553 & 132.0000 & 6.3703 \\
\hline \multirow{3}{*}{$12-18$ yrs } & M & 138.0000 & 3.2071 & 129.7500 & 6.7771 & 133.8750 & 6.6621 \\
\cline { 2 - 8 } & F & 140.2500 & 1.8323 & 132.1250 & 2.8504 & 136.1875 & 4.7919 \\
\cline { 2 - 8 } & T & 139.1250 & 2.7779 & 130.9375 & 5.1700 & 135.0313 & 5.8281 \\
\hline \multirow{3}{*}{ Total } & M & 135.7500 & 3.7683 & 127.9375 & 5.9718 & 131.8437 & 6.3149 \\
\cline { 2 - 8 } & F & 140.1250 & 2.1871 & 130.2500 & 3.5684 & 135.1875 & 5.8001 \\
\cline { 2 - 8 } & T & 137.9375 & 3.7583 & 129.0938 & 4.9797 & 133.5156 & 6.2462 \\
\hline
\end{tabular}

\section{Tests of Between -Subjects Effects}

\footnotetext{
A) $F_{\text {groups }}$ : 85.396; $P<.000(S), F_{\text {ages }}: 10.032 ; P<.002(S), F_{\text {sex }}: 12.208 ; P<.001(S)$

$\mathrm{F}_{\text {groups }} \mathrm{x} \mathrm{F}_{\text {ages }}: .470 ; \mathrm{P}<.496(\mathrm{NS}), \mathrm{F}_{\text {groups }} \mathrm{x} \mathrm{F}_{\text {sex }}: 1.161 ; \mathrm{P}<.286(\mathrm{NS})$

$\mathrm{F}_{\text {ages }} \mathrm{x} \mathrm{F}_{\text {sex }}: 1.161 ; \mathrm{P}<.286(\mathrm{NS}), \mathrm{F}_{\text {groups }} \mathrm{x} \mathrm{F}_{\text {ages }} \mathrm{x} \mathrm{F}_{\text {sex }}: 1.306 ; \mathrm{P}<.258(\mathrm{NS})$
}

Between the groups a significant difference was observed in the mean NSBa values of DS and Normal individuals where DS had higher value $(\mathrm{F}=85.393 ; \mathrm{P}<.000)$. Between age groups also a significant difference was observed $(\mathrm{F}=10.032 ; \mathrm{P}<.002)$ where 12-18 year group had higher value than 6-12 year. Between sex groups also a significant difference was observed $(\mathrm{F}=12.208 ; \mathrm{P}<.001)$ where males had higher NSBa value as compared to females. All the interaction effects were found to be non significant. 
Table 3: Mean SNA values of male and female subjects belonging to different age group in Down's syndrome and Normal groups along with the results of $F$ test

\begin{tabular}{|c|c|c|c|c|c|c|c|}
\hline & Sex & \multicolumn{2}{|l|}{ DS } & \multicolumn{2}{|l|}{ Normal } & \multicolumn{2}{|l|}{ Overall } \\
\hline \multirow{4}{*}{$6-12 \mathrm{yrs}$} & & Mean & S.D & Mean & S.D & Mean & S.D \\
\hline & M & 78.6250 & 5.5016 & 79.8750 & 5.7925 & 79.2500 & 5.4955 \\
\hline & $\mathrm{F}$ & 78.7500 & 4.2003 & 80.6250 & 1.5980 & 79.6875 & 3.2191 \\
\hline & $\mathrm{T}$ & 78.6875 & 4.7289 & 80.2500 & 4.1231 & 79.4688 & 4.4358 \\
\hline \multirow[t]{3}{*}{$12-18$ yrs } & M & 80.2500 & 2.1876 & 81.8750 & 4.1209 & 81.0625 & 3.2958 \\
\hline & $\mathrm{F}$ & 80.7500 & 2.1876 & 81.1250 & 3.1820 & 80.9375 & 2.6450 \\
\hline & $\mathrm{T}$ & 80.5000 & 2.1292 & 81.5000 & 3.5777 & 81.0000 & 2.9403 \\
\hline \multirow[t]{3}{*}{ Total } & M & 79.4375 & 4.1307 & 80.8750 & 4.9649 & 80.1563 & 4.5516 \\
\hline & $\mathrm{F}$ & 79.7500 & 3.3961 & 80.8750 & 2.4461 & 80.3125 & 2.9669 \\
\hline & $\mathrm{T}$ & 79.5938 & 3.7232 & 80.8750 & 3.8500 & 80.2344 & 3.8120 \\
\hline
\end{tabular}

\section{Tests of Between -Subjects Effects}

B) $\mathrm{F}_{\text {groups }}: 1.741 ; \mathrm{P}<.192$ (NS), $\mathrm{F}_{\text {ages }}: 2.487 ; \mathrm{P}<.120$ (NS), $\mathrm{F}_{\text {sex }}: .026 ; \mathrm{P}<.873$ (NS)

$\mathrm{F}_{\text {groups }} \mathrm{X} \mathrm{F}_{\text {ages }}: .084 ; \mathrm{P}<.773$ (NS), $\mathrm{F}_{\text {groups }} \mathrm{x}_{\mathrm{sex}}: .026 ; \mathrm{P}<.873$ (NS)

$\mathrm{F}_{\text {ages }} \mathrm{x} \mathrm{F}_{\text {sex }}: .084 ; \mathrm{P}<.773(\mathrm{NS}), \mathrm{F}_{\text {groups }} \mathrm{x} \mathrm{F}_{\text {ages }} \mathrm{x} \mathrm{F}_{\text {sex }}: .233 ; \mathrm{P}<.631(\mathrm{NS})$

Between the groups, ages and sex non significant differences were observed in the mean SNA values. All the interaction effects were also found to be non significant. 
Table 4: Mean Palpl-NSe values of male and female subjects belonging to different age group in Down's syndrome and Normal groups along with the results of $F$ test

\begin{tabular}{|c|c|c|c|c|c|c|c|}
\hline \multirow{2}{*}{$\begin{array}{l}\begin{array}{l}\text { Age } \\
\text { groups }\end{array} \\
6-12 \text { yrs }\end{array}$} & \multirow[t]{2}{*}{ Sex } & \multicolumn{2}{|l|}{ DS } & \multicolumn{2}{|l|}{ Normal } & \multicolumn{2}{|l|}{ Overall } \\
\hline & & Mean & S.D & Mean & S.D & Mean & S.D \\
\hline & M & 6.1250 & 2.1002 & 7.0000 & 5.1270 & 6.5625 & 3.8117 \\
\hline & $\mathrm{F}$ & 15.9750 & 28.6995 & 5.3750 & 2.3261 & 10.6750 & 20.4172 \\
\hline & $\mathrm{T}$ & 11.0500 & 20.3053 & 6.1875 & 3.9365 & 8.6188 & 14.5980 \\
\hline \multirow[t]{3}{*}{$12-18 \mathrm{yrs}$} & M & 5.7500 & 2.4928 & 6.2500 & 3.0119 & 6.0000 & 2.6833 \\
\hline & $\mathrm{F}$ & 6.6250 & 2.1998 & 7.0000 & 1.7728 & 6.8125 & 1.9397 \\
\hline & $\mathrm{T}$ & 6.1875 & 2.3157 & 6.6250 & 2.4187 & 6.4062 & 2.3398 \\
\hline \multirow[t]{3}{*}{ Total } & M & 5.9375 & 2.2351 & 6.6250 & 4.0804 & 6.2813 & 3.2551 \\
\hline & $\mathrm{F}$ & 11.3000 & 20.2471 & 6.1875 & 2.1670 & 8.7437 & 14.4006 \\
\hline & $\mathrm{T}$ & 8.6187 & 14.4291 & 6.4062 & 3.2215 & 7.5125 & 10.4306 \\
\hline
\end{tabular}

Tests of Between- Subjects Effects

C) $\mathrm{F}_{\text {groups }}: .710 ; \mathrm{P}<.403$ (NS), $\mathrm{F}_{\text {ages }}: .710 ; \mathrm{P}<.403$ (NS), $\mathrm{F}_{\text {sex }}: .879 ; \mathrm{P}<.353$ (NS)

$\mathrm{F}_{\text {groups }} \mathrm{X} \mathrm{F}_{\text {ages }}: .1 .018 ; \mathrm{P}<.317(\mathrm{NS}), \mathrm{F}_{\text {groups }} \mathrm{X} \mathrm{F}_{\text {sex }}: 1.219 ; \mathrm{P}<.274(\mathrm{NS})$

$\mathrm{F}_{\text {ages }} \mathrm{x} \mathrm{F}_{\text {sex }}: .395 ; \mathrm{P}<.532(\mathrm{NS}), \mathrm{F}_{\text {groups }} \mathrm{x} \mathrm{F}_{\text {ages }} \mathrm{x} \mathrm{F}_{\text {sex }}: 1.167 ; \mathrm{P}<.285$ (NS)

Between the groups, ages and sex non significant differences were observed in the mean Palpl-NSe values. All the interaction effects were also found to be non significant. 
Table 5: Mean U1-NA( $\left(^{0}\right)$ values of male and female subjects belonging to different age group in Down's syndrome and Normal groups along with the results of $F$ test

\begin{tabular}{|c|c|c|c|c|c|c|c|}
\hline \multirow{2}{*}{$\begin{array}{l}\begin{array}{l}\text { Age } \\
\text { groups }\end{array} \\
6-12 \text { yrs }\end{array}$} & \multirow[t]{2}{*}{ Sex } & \multicolumn{2}{|l|}{ DS } & \multicolumn{2}{|l|}{ Normal } & \multicolumn{2}{|l|}{ Overall } \\
\hline & & Mean & S.D & Mean & S.D & Mean & S.D \\
\hline & $\mathrm{M}$ & 22.7500 & 3.9551 & 25.1250 & 10.5212 & 23.9375 & 7.7758 \\
\hline & F & 37.2500 & 5.4968 & 24.3750 & 6.7175 & 30.8125 & 8.9086 \\
\hline & $\mathrm{T}$ & 30.0000 & 8.8015 & 24.7500 & 8.5362 & 27.3750 & 8.9362 \\
\hline \multirow[t]{3}{*}{$12-18 \mathrm{yrs}$} & M & 35.6250 & 7.5958 & 24.1250 & 3.4821 & 29.8750 & 8.2371 \\
\hline & $\mathrm{F}$ & 39.1250 & 3.8707 & 28.5000 & 13.6172 & 33.8125 & 11.1189 \\
\hline & $\mathrm{T}$ & 37.3750 & 6.0978 & 26.3125 & 9.8639 & 31.8438 & 9.8312 \\
\hline \multirow[t]{3}{*}{ Total } & M & 29.1875 & 8.8560 & 24.6250 & 7.5884 & 26.9063 & 8.4371 \\
\hline & $\mathrm{F}$ & 38.1875 & 4.6935 & 26.4375 & 10.5891 & 32.3125 & 10.0272 \\
\hline & $\mathrm{T}$ & 33.6875 & 8.3374 & 25.5313 & 9.1086 & 29.6094 & 9.5877 \\
\hline
\end{tabular}

D) Tests of Between -Subjects Effects

E) $F_{\text {groups }}: 18.044 ; P<.000(S), F_{\text {ages }}: 5.417 ; P<.024(S), F_{\text {sex }}: 7.928 ; P<.007(S)$

$\mathrm{F}_{\text {groups }} \mathrm{x} \mathrm{F}_{\text {ages }}: 2.291 ; \mathrm{P}<.136(\mathrm{NS}), \mathrm{F}_{\text {groups }} \mathrm{x} \mathrm{F}_{\text {sex }}: 3.503 ; \mathrm{P}<.066(\mathrm{NS})$

$\mathrm{F}_{\text {ages }} \mathrm{x} \mathrm{F}_{\text {sex }}: .585 ; \mathrm{P}<.448(\mathrm{NS}), \mathrm{F}_{\text {groups }} \mathrm{x} \mathrm{F}_{\text {ages }} \mathrm{x} \mathrm{F}_{\text {sex }}: 4.408 ; \mathrm{P}<.040(\mathrm{~S})$

Between the groups a significant difference was observed in the mean U1-NA( $\left(^{0}\right)$ values of DS and Normal individual where DS had higher value $(\mathrm{F}=18.044 ; \mathrm{P}<.000)$. Between age groups also a significant difference was observed $(\mathrm{F}=18.044 ; \mathrm{P}<.024)$ where $12-18$ year group had higher value than 6-12 year Between sex groups also a significant difference was observed $(\mathrm{F}=7.928 ; \mathrm{P}<.007)$ where females had higher U1-NA $\left(^{0}\right)$ value as compared to males. In 6-12 years normal group females had lower value than males whereas in others females had higher value than males. All the other interaction effects were found to be non significant. 
Table 6: Mean U1-SN values of male and female subjects belonging to different age group in Down's syndrome and Normal groups along with the results of $F$ test

\begin{tabular}{|c|c|c|c|c|c|c|c|}
\hline \multirow{2}{*}{$\begin{array}{l}\begin{array}{l}\text { Age } \\
\text { groups }\end{array} \\
6-12 \mathrm{yrs}\end{array}$} & \multirow[t]{2}{*}{ Sex } & \multicolumn{2}{|l|}{ DS } & \multicolumn{2}{|l|}{ Normal } & \multicolumn{2}{|l|}{ Overall } \\
\hline & & Mean & S.D & Mean & S.D & Mean & S.D \\
\hline & M & 102.2500 & 6.5629 & 105.3750 & 10.0561 & 103.8125 & 8.3604 \\
\hline & $\mathrm{F}$ & 112.5000 & 9.5019 & 105.3750 & 7.5012 & 108.9375 & 9.0515 \\
\hline & $\mathrm{T}$ & 107.3750 & 9.5000 & 105.3750 & 8.5703 & 106.3750 & 8.9578 \\
\hline \multirow[t]{3}{*}{$12-18 \mathrm{yrs}$} & M & 118.6250 & 8.5513 & 106.6250 & 6.5452 & 112.6250 & 9.6186 \\
\hline & $\mathrm{F}$ & 119.7500 & 4.5277 & 104.1250 & 6.5124 & 111.9375 & 9.7192 \\
\hline & $\mathrm{T}$ & 119.1875 & 6.6354 & 105.3750 & 6.4382 & 112.2813 & 9.5182 \\
\hline \multirow[t]{3}{*}{ Total } & $\mathrm{M}$ & 110.4375 & 11.2129 & 106.0000 & 8.2219 & 108.2188 & 9.9312 \\
\hline & $\mathrm{F}$ & 116.1250 & 8.1066 & 104.7500 & 6.8166 & 110.4375 & 9.3634 \\
\hline & $\mathrm{T}$ & 113.2813 & 10.0490 & 105.3750 & 7.4563 & 109.3281 & 9.6396 \\
\hline
\end{tabular}

\title{
Tests of Between -Subjects Effects
}

\author{
F) $F_{\text {groups }}: 17.037 ; P<.000(S), F_{\text {ages }}: 9.508 ; P<.003(S), F_{\text {sex }}: 1.342 ; P<.252$ (NS) \\ $\mathrm{F}_{\text {groups }} \mathrm{x} \mathrm{F}_{\text {ages }}: 9.508 ; \mathrm{P}<.003(\mathrm{~S}), \mathrm{F}_{\text {groups }} \mathrm{x} \mathrm{F}_{\text {sex }}: 3.279 ; \mathrm{P}<.076(\mathrm{NS})$ \\ $\mathrm{F}_{\text {ages }} \mathrm{x} \mathrm{F}_{\text {sex }}: 2.302 ; \mathrm{P}<.135$ (NS), $\mathrm{F}_{\text {groups }} \mathrm{x}_{\mathrm{F}_{\text {ages }} \mathrm{x}} \mathrm{F}_{\text {sex }}: .748 ; \mathrm{P}<.391$ (NS)
}

Between the groups a significant difference was observed in the mean U1-SN values of DS and Normal individual where DS had higher value $(\mathrm{F}=17.037 ; \mathrm{P}<.000)$. Between age groups also a significant difference was observed $(\mathrm{F}=9.508 ; \mathrm{P}<.003)$ where $12-18$ year group had higher value than 6-12 year. Between sex groups a non significant difference was observed $(\mathrm{F}=1.342 ; \mathrm{P}<252)$. There was a significant difference in the mean value of 12-18 year of DS and Normal as compared to 6-12 year of DS and Normal. In DS group the values were higher in 12-18 year age than 6-12 year, whereas values were almost same in Normals. All the other interaction effects were found to be non significant. 
Table 7: Mean U1-Palpl values of male and female subjects belonging to different age group in Down's syndrome and Normal groups along with the results of $F$ test

\begin{tabular}{|l|l|l|l|l|l|l|l|}
\hline \multirow{2}{*}{$\begin{array}{l}\text { Age } \\
\text { groups }\end{array}$} & \multirow{2}{*}{ Sex } & \multicolumn{2}{l}{ DS } & \multicolumn{2}{l}{ Normal } & \multicolumn{2}{l|}{ Overall } \\
\hline \multirow{3}{*}{ 6-12 yrs } & & Mean & S.D & Mean & S.D & Mean & S.D \\
\cline { 2 - 8 } & M & 111.5000 & 5.5291 & 113.3750 & 7.7448 & 112.4375 & 6.5724 \\
\cline { 2 - 8 } & F & 123.0000 & 6.7401 & 119.0000 & 7.3095 & 121.0000 & 7.0993 \\
\cline { 2 - 8 } & T & 117.2500 & 8.4103 & 116.1875 & 7.8334 & 116.7187 & 8.0130 \\
\hline \multirow{5}{*}{$12-18$ yrs } & M & 127.5000 & 9.4567 & 117.5000 & 6.9898 & 122.5000 & 9.5499 \\
\cline { 2 - 8 } & F & 129.1250 & 3.3991 & 113.1250 & 4.2237 & 121.1250 & 9.0545 \\
\cline { 2 - 8 } & T & 128.3125 & 6.9159 & 115.3125 & 6.0191 & 121.8125 & 9.1808 \\
\hline \multirow{3}{*}{ Total } & M & 119.5000 & 11.1475 & 115.4375 & 7.4384 & 117.4688 & 9.5478 \\
\cline { 2 - 8 } & F & 126.0625 & 6.0494 & 116.0625 & 6.5163 & 121.0625 & 8.0038 \\
\cline { 2 - 8 } & T & 122.7813 & 9.4314 & 115.7500 & 6.8862 & 119.2656 & 8.9252 \\
\hline
\end{tabular}

\section{Tests of Between -Subjects Effects}

G) $\mathrm{F}_{\text {groups }}: 17.721 ; \mathrm{P}<.000(\mathrm{~S}), \mathrm{F}_{\text {ages }}: 9.301 ; \mathrm{P}<.003(\mathrm{~S}), \mathrm{F}_{\text {sex }}: 4.629 ; \mathrm{P}<.036(\mathrm{~S})$

$\mathrm{F}_{\text {groups }} \mathrm{x} \mathrm{F}_{\text {ages }}: 12.770 ; \mathrm{P}<.001(\mathrm{~S}), \mathrm{F}_{\text {groups }} \mathrm{x} \mathrm{F}_{\text {sex }}: 3.159 ; \mathrm{P}<.081(\mathrm{NS})$

$\mathrm{F}_{\text {ages }} \mathrm{x} \mathrm{F}_{\text {sex }}: 8.850 ; \mathrm{P}<.004(\mathrm{~S}), \mathrm{F}_{\text {groups }} \mathrm{x} \mathrm{F}_{\text {ages }} \mathrm{x} \mathrm{F}_{\text {sex }}: .000 ; \mathrm{P}<.2985(\mathrm{NS})$

Between the groups a significant difference was observed in the mean U1-Palpl a values of DS and Normal individual where DS had higher value $(\mathrm{F}=17.721 ; \mathrm{P}<.000)$. Between age groups also a significant difference was observed $(\mathrm{F}=9.301 ; \mathrm{P}<.003)$ where $12-18$ year group had higher value than 612 year. Between sex groups also a significant difference was observed $(\mathrm{F}=4.629 ; \mathrm{P}<.036)$ where females had higher U1-Palpl value as compared to males.

There was a significant difference in the mean value of 12-18 year of DS and Normal as compared to 6-12 year of DS and Normal $(\mathrm{F}=12.770 ; \mathrm{P}<.001)$. In DS group the values are higher in 1218 year age than 6-12 year, whereas values are almost same in Normal.

There was a significant difference in the mean value of 12-18 year of males and females as compared to 6-12 year of males and females $(\mathrm{F}=18.850 ; \mathrm{P}<.004)$. In 6-12 year group females had higher value than males, whereas in 12-18 year group males had higher value than females. 
Table 8: Mean SN values of male and female subjects belonging to different age group in Down's syndrome and Normal groups along with the results of $F$ test

\begin{tabular}{|c|c|c|c|c|c|c|c|}
\hline \multirow{2}{*}{$\begin{array}{l}\begin{array}{l}\text { Age } \\
\text { groups }\end{array} \\
6-12 \text { yrs }\end{array}$} & \multirow[t]{2}{*}{ Sex } & \multicolumn{2}{|l|}{ DS } & \multicolumn{2}{|l|}{ Normal } & \multicolumn{2}{|l|}{ Overall } \\
\hline & & Mean & S.D & Mean & S.D & Mean & S.D \\
\hline & $\mathrm{M}$ & 60.0000 & 2.6726 & 67.6250 & 3.0208 & 63.8125 & 4.8058 \\
\hline & F & 59.1250 & 2.7999 & 66.0000 & 1.8516 & 62.5625 & 4.2264 \\
\hline & $\mathrm{T}$ & 59.5625 & 2.6825 & 66.8125 & 2.5617 & 63.1875 & 4.4969 \\
\hline \multirow[t]{3}{*}{$12-18 \mathrm{yrs}$} & M & 64.6250 & 3.8149 & 70.8750 & 3.0443 & 67.7500 & 4.6404 \\
\hline & $\mathrm{F}$ & 60.7500 & 2.5495 & 67.8750 & 3.3991 & 64.3125 & 4.6864 \\
\hline & $\mathrm{T}$ & 62.6875 & 3.7188 & 69.3750 & 3.4809 & 66.0313 & 4.9087 \\
\hline \multirow[t]{3}{*}{ Total } & $\mathrm{M}$ & 62.3125 & 3.9786 & 69.2500 & 3.3764 & 65.7813 & 5.0592 \\
\hline & $\mathrm{F}$ & 59.9375 & 2.7195 & 66.9375 & 2.8159 & 63.4375 & 4.4789 \\
\hline & $\mathrm{T}$ & 61.1250 & 3.5628 & 68.0937 & 3.2761 & 64.6094 & 4.8848 \\
\hline
\end{tabular}

\section{Tests of Between -Subjects Effects}

H) $\mathrm{F}_{\text {groups }}$ : 89.556; $\mathrm{P}<.000(\mathrm{~S}), \mathrm{F}_{\text {ages }}: 14.913 ; \mathrm{P}<.000(\mathrm{~S}), \mathrm{F}_{\text {sex }}: 10.130 ; \mathrm{P}<.002(\mathrm{~S})$

$\mathrm{F}_{\text {groups }} \mathrm{x} \mathrm{F}_{\text {ages }}: .146 ; \mathrm{P}<.704(\mathrm{NS}), \mathrm{F}_{\text {groups }} \mathrm{x}_{\mathrm{sex}}: .002 ; \mathrm{P}<.966(\mathrm{NS})$

$\mathrm{F}_{\text {ages }} \mathrm{x} \mathrm{F}_{\text {sex }}: 2.206 ; \mathrm{P}<.143(\mathrm{NS}), \mathrm{F}_{\text {groups }} \mathrm{x}_{\mathrm{F}_{\text {ages }} \mathrm{x}} \mathrm{F}_{\text {sex }}: .34 ; \mathrm{P}<.583(\mathrm{NS})$

I) Between the groups a significant difference was observed in the mean SN values of DS and Normal individual where $\mathrm{DS}$ had higher value $(\mathrm{F}=89.556 ; \mathrm{P}<.000)$. Between age groups also a significant difference was observed $(\mathrm{F}=14.913 ; \mathrm{P}<.000)$ where 12-18 year group had higher value than 6-12 year. Between sex groups also a significant difference was observed $(\mathrm{F}=10.130 ; \mathrm{P}<.002)$ where male had higher SN value as compared to female. All the interaction effects were found to be non significant. 
Table 9: Mean S-Ba values of male and female subjects belonging to different age group in Down's syndrome and Normal groups along with the results of $F$ test

\begin{tabular}{|c|c|c|c|c|c|c|c|}
\hline \multirow{2}{*}{\begin{tabular}{|l|}
$\begin{array}{l}\text { Age } \\
\text { groups }\end{array}$ \\
$6-12 \mathrm{yrs}$ \\
\end{tabular}} & \multirow[t]{2}{*}{ Sex } & \multicolumn{2}{|l|}{ DS } & \multicolumn{2}{|l|}{ Normal } & \multicolumn{2}{|l|}{ Overall } \\
\hline & & Mean & S.D & Mean & S.D & Mean & S.D \\
\hline & M & 42.3750 & 2.6152 & 43.2500 & 1.8323 & 42.8125 & 2.2277 \\
\hline & $\mathrm{F}$ & 41.5000 & 4.3425 & 42.5000 & 1.8516 & 42.0000 & 3.2660 \\
\hline & $\mathrm{T}$ & 41.9375 & 3.4923 & 42.8750 & 1.8212 & 42.4063 & 2.7808 \\
\hline \multirow[t]{3}{*}{$12-18$ yrs } & M & 44.2500 & 3.1053 & 48.2500 & 3.9551 & 46.2500 & 4.0083 \\
\hline & $\mathrm{F}$ & 41.7500 & 1.8323 & 45.0000 & 1.8516 & 43.3750 & 2.4461 \\
\hline & $\mathrm{T}$ & 43.0000 & 2.7809 & 46.6250 & 3.4230 & 44.8125 & 3.5780 \\
\hline \multirow[t]{3}{*}{ Total } & M & 43.3125 & 2.9375 & 45.7500 & 3.9412 & 44.5313 & 3.6366 \\
\hline & $\mathrm{F}$ & 41.6250 & 3.2223 & 43.7500 & 2.2061 & 42.6875 & 2.9231 \\
\hline & $\mathrm{T}$ & 42.4688 & 3.1519 & 44.7500 & 3.3020 & 43.6094 & 3.4022 \\
\hline
\end{tabular}

\section{Tests of Between -Subjects Effects}

\section{J) $\quad F_{\text {groups }}: 10.319 ; P<.002(S), F_{\text {ages }}: 11.481 ; P<.001(S), F_{\text {sex }}: 6.741 ; P<.012(S)$}

$\mathrm{F}_{\text {groups }} \mathrm{x} \mathrm{F}_{\text {ages }}: 3.580 ; \mathrm{P}<.064(\mathrm{NS}), \mathrm{F}_{\text {groups }} \mathrm{x} \mathrm{F}_{\text {sex }}: 048 ; \mathrm{P}<.827(\mathrm{NS})$

$\mathrm{F}_{\text {ages }} \mathrm{x} \mathrm{F}_{\text {sex }}: 2.109 ; \mathrm{P}<.152(\mathrm{NS}), \mathrm{F}_{\text {groups }} \mathrm{x} \mathrm{F}_{\text {ages }} \mathrm{x} \mathrm{F}_{\text {sex }}: .095 ; \mathrm{P}<.759$ (NS)

Between the groups a significant difference was observed in the mean S-Ba values of DS and Normal individual where $\mathrm{DS}$ had higher value $(\mathrm{F}=10.319 ; \mathrm{P}<.002)$. Between age groups also a significant difference was observed $(\mathrm{F}=11.481 ; \mathrm{P}<.001)$ where $12-18$ year group had higher value than 6-12 year. Between sex groups also a significant difference was observed $(\mathrm{F}=6.741 ; \mathrm{P}<.012)$ where male had higher $\mathrm{S}$-Ba value as compared to female. All the interaction effects were found to be non significant. 
Table 10: Mean Ba-N values of male and female subjects belonging to different age group in Down's syndrome and Normal groups along with the results of $F$ test

\begin{tabular}{|c|c|c|c|c|c|c|c|}
\hline \multirow{2}{*}{$\begin{array}{l}\begin{array}{l}\text { Age } \\
\text { groups }\end{array} \\
6-12 \text { yrs }\end{array}$} & \multirow[t]{2}{*}{ Sex } & \multicolumn{2}{|l|}{ DS } & \multicolumn{2}{|l|}{ Normal } & \multicolumn{2}{|l|}{ Overall } \\
\hline & & Mean & S.D & Mean & S.D & Mean & S.D \\
\hline & $\mathrm{M}$ & 94.7500 & 3.3700 & 99.2500 & 4.7434 & 97.0000 & 4.6043 \\
\hline & $\mathrm{F}$ & 96.1250 & 3.5632 & 98.1250 & 3.5229 & 97.1250 & 3.5754 \\
\hline & $\mathrm{T}$ & 95.4375 & 3.4248 & 98.6875 & 4.0779 & 97.0625 & 4.0556 \\
\hline \multirow[t]{3}{*}{$12-18$ yrs } & M & 102.0000 & 5.3184 & 108.1250 & 5.3033 & 105.0625 & 6.0274 \\
\hline & $\mathrm{F}$ & 97.5000 & 3.8914 & 93.7500 & 33.7586 & 95.6250 & 23.2948 \\
\hline & $\mathrm{T}$ & 99.7500 & 5.0662 & 100.9375 & 24.4962 & 100.3437 & 17.4108 \\
\hline \multirow[t]{3}{*}{ Total } & M & 98.3750 & 5.7023 & 103.6875 & 6.6805 & 101.0313 & 6.6792 \\
\hline & $\mathrm{F}$ & 96.8125 & 3.6737 & 95.9375 & 23.2965 & 96.3750 & 16.4115 \\
\hline & $\mathrm{T}$ & 97.5938 & 4.7848 & 99.8125 & 17.3120 & 98.7031 & 12.6487 \\
\hline
\end{tabular}

Tests of Between -Subjects Effects

$\mathrm{F}_{\text {groups }}: .496 ; \mathrm{P}<.484(\mathrm{NS}), \mathrm{F}_{\text {ages }}: 1.085 ; \mathrm{P}<.302(\mathrm{NS}), \mathrm{F}_{\text {sex }}: 2.185 ; \mathrm{P}<.145(\mathrm{NS})$

$\mathrm{F}_{\text {groups }} \mathrm{x} \mathrm{F}_{\text {ages }}: .107 ; \mathrm{P}<.745(\mathrm{NS}), \mathrm{F}_{\text {groups }} \mathrm{x} \mathrm{F}_{\text {sex }}: .965 ; \mathrm{P}<.330(\mathrm{NS})$

$\mathrm{F}_{\text {ages }} \mathrm{x} \mathrm{F}_{\text {sex }}: 2.304 ; \mathrm{P}<.135(\mathrm{NS}), \mathrm{F}_{\text {groups }} \mathrm{x} \mathrm{F}_{\text {ages }} \mathrm{x} \mathrm{F}_{\text {sex }}: .343 ; \mathrm{P}<.561(\mathrm{NS})$

Between the groups, ages and sex a non significant differences were observed in the mean Palpl-NSe values. All the interaction effects were also found to be non significant. 
Table 11: Mean N-ANS values of male and female subjects belonging to different age group in Down's syndrome and Normal groups along with the results of $F$ test

\begin{tabular}{|c|c|c|c|c|c|c|c|}
\hline \multirow{2}{*}{$\begin{array}{l}\begin{array}{l}\text { Age } \\
\text { groups }\end{array} \\
6-12 \text { yrs }\end{array}$} & \multirow[t]{2}{*}{ Sex } & \multicolumn{2}{|l|}{ DS } & \multicolumn{2}{|l|}{ Normal } & \multicolumn{2}{|l|}{ Overall } \\
\hline & & Mean & S.D & Mean & S.D & Mean & S.D \\
\hline & $\mathrm{M}$ & 44.1250 & 3.9438 & 45.0000 & 3.8914 & 44.5625 & 3.8117 \\
\hline & F & 42.3750 & 2.9731 & 45.6250 & 5.2082 & 44.0000 & 4.4272 \\
\hline & $\mathrm{T}$ & 43.2500 & 3.4928 & 45.3125 & 4.4530 & 44.2813 & 4.0738 \\
\hline \multirow[t]{3}{*}{$12-18 \mathrm{yrs}$} & M & 48.5000 & 2.6186 & 52.5000 & 2.0000 & 50.5000 & 3.0551 \\
\hline & $\mathrm{F}$ & 44.2500 & 2.8158 & 51.7500 & 2.9641 & 48.0000 & 4.7749 \\
\hline & $\mathrm{T}$ & 46.3750 & 3.4230 & 52.1250 & 2.4732 & 49.2500 & 4.1426 \\
\hline \multirow[t]{3}{*}{ Total } & $\mathrm{M}$ & 46.3125 & 3.9449 & 48.7500 & 4.8922 & 47.5313 & 4.5436 \\
\hline & $\mathrm{F}$ & 43.3125 & 2.9602 & 48.6875 & 5.1732 & 46.0000 & 4.9644 \\
\hline & $\mathrm{T}$ & 44.8125 & 3.7540 & 48.7188 & 4.9529 & 46.7656 & 4.7834 \\
\hline
\end{tabular}

\section{Tests of Between -Subjects Effects}

$\mathrm{F}_{\text {groups }}: 20.727 ; \mathrm{P}<.000(\mathrm{~S}), \mathrm{F}_{\text {ages }}: 33.536 ; \mathrm{P}<.000(\mathrm{~S}), \mathrm{F}_{\text {sex }}: 3.185 ; \mathrm{P}<.080(\mathrm{~S})$

$\mathrm{F}_{\text {groups }} \mathrm{x} \mathrm{F}_{\text {ages }}: 4.618 ; \mathrm{P}<.036(\mathrm{~S}), \mathrm{F}_{\text {groups }} \mathrm{x} \mathrm{F}_{\text {sex }}: 2.930 ; \mathrm{P}<.092(\mathrm{NS})$

$\mathrm{F}_{\text {ages }} \mathrm{x} \mathrm{F}_{\text {sex }}: 1.275 ; \mathrm{P}<.264(\mathrm{NS}), \mathrm{F}_{\text {groups }} \mathrm{x} \mathrm{F}_{\text {ages }} \mathrm{x} \mathrm{F}_{\text {sex }}: .107 ; \mathrm{P}<.744(\mathrm{NS})$

Between the groups a significant difference was observed in the mean N-ANS values of DS and Normal individual where $\mathrm{DS}$ had lower value $(\mathrm{F}=20.727 ; \mathrm{P}<.000)$. Between age groups also a significant difference was observed $(\mathrm{F}=33.536 ; \mathrm{P}<.000)$ where $12-18$ year group had higher value than $6-12$ year. Between sex groups a non significant difference was observed $(\mathrm{F}=3.185 ; \mathrm{P}<.080)$. There is a significant difference in the mean value of 12-18 year of DS and Normal as compared to 6-12 year of DS and Normal $(\mathrm{F}=.4 .618 ; \mathrm{P}<.036)$. In Normal group the value was much higher in 12-18 group than 6-12 year group as compared to that of DS. All the other interaction effects were found to be non significant. 
Table 12: Mean PNS-ANS values of male and female subjects belonging to different age group in Down's syndrome and Normal groups along with the results of $F$ test

\begin{tabular}{|c|c|c|c|c|c|c|c|}
\hline \multirow{2}{*}{$\begin{array}{l}\begin{array}{l}\text { Age } \\
\text { groups }\end{array} \\
6-12 \text { yrs }\end{array}$} & \multirow[t]{2}{*}{ Sex } & \multicolumn{2}{|l|}{ DS } & \multicolumn{2}{|l|}{ Normal } & \multicolumn{2}{|l|}{ Overall } \\
\hline & & Mean & S.D & Mean & S.D & Mean & S.D \\
\hline & $\mathrm{M}$ & 40.7500 & 1.3887 & 45.5000 & 2.2678 & 43.1250 & 3.0523 \\
\hline & F & 38.1250 & 2.7999 & 47.2500 & 2.9641 & 42.6875 & 5.4738 \\
\hline & $\mathrm{T}$ & 39.4375 & 2.5290 & 46.3750 & 2.7049 & 42.9063 & 4.3653 \\
\hline \multirow[t]{3}{*}{$12-18 \mathrm{yrs}$} & M & 44.5000 & 2.4495 & 54.7500 & 2.7646 & 49.6250 & 5.8637 \\
\hline & $\mathrm{F}$ & 42.6250 & 3.2043 & 51.5000 & 3.3806 & 47.0625 & 5.5794 \\
\hline & $\mathrm{T}$ & 43.5625 & 2.9205 & 53.1250 & 3.4230 & 48.3438 & 5.7788 \\
\hline \multirow[t]{3}{*}{ Total } & $\mathrm{M}$ & 42.6250 & 2.7295 & 50.1250 & 5.3650 & 46.3750 & 5.6611 \\
\hline & $\mathrm{F}$ & 40.3750 & 3.7216 & 49.3750 & 3.7749 & 44.8750 & 5.8737 \\
\hline & $\mathrm{T}$ & 41.5000 & 3.4078 & 49.7500 & 4.5791 & 45.6250 & 5.7721 \\
\hline
\end{tabular}

\section{Tests of Between -Subjects Effects}

$\mathrm{F}_{\text {groups: }}: 147.572 ; \mathrm{P}<.000(\mathrm{~S}), \mathrm{F}_{\text {ages }}: 64.105 ; \mathrm{P}<.000(\mathrm{~S}), \mathrm{F}_{\text {sex }}: 4.878 ; \mathrm{P}<.031(\mathrm{~S})$

$\mathrm{F}_{\text {groups }} \mathrm{x} \mathrm{F}_{\text {ages }}: 3.735 ; \mathrm{P}<.058(\mathrm{NS}), \mathrm{F}_{\text {groups }} \mathrm{x} \mathrm{F}_{\text {sex }}: 1.220 ; \mathrm{P}<.274(\mathrm{NS})$

$\mathrm{F}_{\text {ages }} \mathrm{x} \mathrm{F}_{\text {sex }}: 2.448 ; \mathrm{P}<.123(\mathrm{NS}), \mathrm{F}_{\text {groups }} \mathrm{x} \mathrm{F}_{\text {ages }} \mathrm{x} \mathrm{F}_{\text {sex }}: 4.480 ; \mathrm{P}<.039(\mathrm{~S})$

Between the groups a significant difference was observed in the mean PNS-ANS values of DS and Normal individual where Normal had higher value $(\mathrm{F}=147.572 ; \mathrm{P}<.000)$. Between age groups also a significant difference was observed $(\mathrm{F}=64.105 ; \mathrm{P}<.000)$ where $12-18$ year group had higher value than 612 year. Between sex groups also a significant difference was observed $(\mathrm{F}=4.878 ; \mathrm{P}<.031)$ where male had higher PNS-ANS value as compared to female. 
Table 13: Mean U1-NA(mm) values of male and female subjects belonging to different age group in Down's syndrome and Normal groups along with the results of $F$ test

\begin{tabular}{|c|c|c|c|c|c|c|c|}
\hline Age & Sex & \multicolumn{2}{|l|}{ DS } & \multicolumn{2}{|c|}{ Normal } & \multicolumn{2}{|l|}{ Overall } \\
\hline \multirow[t]{4}{*}{$6-12 \mathrm{yrs}$} & & Mean & S.D & Mean & S.D & Mean & S.D \\
\hline & M & 4.1875 & 1.3076 & 5.7500 & 4.5277 & 4.9688 & 3.3190 \\
\hline & F & 9.0000 & 2.3905 & 4.8750 & 2.7484 & 6.9375 & 3.2755 \\
\hline & $\mathrm{T}$ & 6.5937 & 3.1049 & 5.3125 & 3.6463 & 5.9531 & 3.3944 \\
\hline \multirow[t]{3}{*}{$12-18 \mathrm{yrs}$} & M & 8.0000 & 4.6599 & 5.0000 & 1.5119 & 6.5000 & 3.6878 \\
\hline & $\mathrm{F}$ & 10.2500 & 3.0119 & 4.2500 & 2.4928 & 7.2500 & 4.0906 \\
\hline & $\mathrm{T}$ & 9.1250 & 3.9644 & 4.6250 & 2.0290 & 6.8750 & 3.8500 \\
\hline \multirow[t]{3}{*}{ Total } & M & 6.0938 & 3.8480 & 5.3750 & 3.2838 & 5.7344 & 3.5378 \\
\hline & $\mathrm{F}$ & 9.6250 & 2.7049 & 4.5625 & 2.5552 & 7.0937 & 3.6488 \\
\hline & $\mathrm{T}$ & 7.8594 & 3.7314 & 4.9688 & 2.9236 & 6.4141 & 3.6303 \\
\hline
\end{tabular}

Tests of Between -Subjects Effects

$\mathrm{F}_{\text {groups }}: 14.306 ; \mathrm{P}<.000(\mathrm{~S}), \mathrm{F}_{\text {ages }}: 1.455 ; \mathrm{P}<.233(\mathrm{NS}), \mathrm{F}_{\text {sex }}: 3.164 ; \mathrm{P}<.081(\mathrm{~S})$

$\mathrm{F}_{\text {groups }} \mathrm{x} \mathrm{F}_{\text {ages }}: 4.434 ; \mathrm{P}<.040(\mathrm{~S}), \mathrm{F}_{\text {groups }} \mathrm{x} \mathrm{F}_{\text {sex }}: 8.076 ; \mathrm{P}<.006(\mathrm{~S})$

$\mathrm{F}_{\text {ages }} \mathrm{x} \mathrm{F}_{\text {sex }}: .636 ; \mathrm{P}<.429(\mathrm{NS}), \mathrm{F}_{\text {groups }} \mathrm{x} \mathrm{F}_{\text {ages }} \mathrm{x} \mathrm{F}_{\text {sex }}: .773 ; \mathrm{P}<.383$ (NS)

Between the groups a significant difference was observed in the mean U1-NA(mm) a values of DS and Normal individual where DS had higher value $(\mathrm{F}=14.306 ; \mathrm{P}<.000)$.

Between age groups also a significant difference was observed $(\mathrm{F}=1.455 ; \mathrm{P}<.233)$ where $12-18$ year group had higher value than 6-12 year. Between sex groups a non significant difference was observed $(\mathrm{F}=3.164 ; \mathrm{P}<.081)$.

There is a significant difference in the mean value of 12-18 year of DS and Normal as compared to 6-12 year of DS and Normal $(\mathrm{F}=4.434 ; \mathrm{P}<.040)$. In DS group the values are higher in 12-18 year age than 6-12 year, whereas values are almost same in Normals.

There is a significant difference in the mean value of male and female of DS as compared to male and female of Normal $(\mathrm{F}=8.076 ; \mathrm{P}<.006)$. In $\mathrm{DS}$ group females had higher value than males whereas in Normal males had higher value than females. 
Table 14:Mean Palpl-U1 values of male and female subjects belonging to different age group in Down's syndrome and Normal groups along with the results of $F$ test

\begin{tabular}{|c|c|c|c|c|c|c|c|}
\hline \multirow{2}{*}{$\begin{array}{l}\begin{array}{l}\text { Age } \\
\text { groups }\end{array} \\
6-12 \text { yrs }\end{array}$} & \multirow[t]{2}{*}{ Sex } & \multicolumn{2}{|l|}{ DS } & \multicolumn{2}{|l|}{ Normal } & \multicolumn{2}{|l|}{ Overall } \\
\hline & & Mean & S.D & Mean & S.D & Mean & S.D \\
\hline & $\mathrm{M}$ & 20.5000 & 2.1381 & 24.2500 & 2.7124 & 22.3750 & 3.0523 \\
\hline & $\mathrm{F}$ & 19.7500 & 1.6690 & 24.2500 & 1.8323 & 22.0000 & 2.8752 \\
\hline & $\mathrm{T}$ & 20.1250 & 1.8930 & 24.2500 & 2.2361 & 22.1875 & 2.9231 \\
\hline \multirow[t]{3}{*}{$12-18 \mathrm{yrs}$} & M & 23.0000 & 3.3381 & 31.5000 & 2.9761 & 27.2500 & 5.3479 \\
\hline & $\mathrm{F}$ & 22.3750 & 5.1530 & 26.7500 & 5.3117 & 24.5625 & 5.5374 \\
\hline & $\mathrm{T}$ & 22.6875 & 4.2066 & 29.1250 & 4.8287 & 25.9062 & 5.5262 \\
\hline \multirow[t]{3}{*}{ Total } & $\mathrm{M}$ & 21.7500 & 3.0000 & 27.8750 & 4.6458 & 24.8125 & 4.9477 \\
\hline & $\mathrm{F}$ & 21.0625 & 3.9407 & 25.5000 & 4.0497 & 23.2813 & 4.5311 \\
\hline & $\mathrm{T}$ & 21.4063 & 3.4628 & 26.6875 & 4.4536 & 24.0469 & 4.7690 \\
\hline
\end{tabular}

\section{Tests of Between -Subjects Effects}

$\mathrm{F}_{\text {groups }}: 38.455 ; \mathrm{P}<.000(\mathrm{~S}), \mathrm{F}_{\text {ages }}: 19.067 ; \mathrm{P}<.000(\mathrm{~S}), \mathrm{F}_{\text {sex }}: 3.233 ; \mathrm{P}<.078$ (NS)

$\mathrm{F}_{\text {groups }} \mathrm{x} \mathrm{F}_{\text {ages }}: 1.843 ; \mathrm{P}<.80(\mathrm{NS}), \mathrm{F}_{\text {groups }} \mathrm{x} \mathrm{F}_{\text {sex }}: .982 ; \mathrm{P}<.326(\mathrm{NS})$

$\mathrm{F}_{\text {ages }} \mathrm{x} \mathrm{F}_{\text {sex }}: 1.843 ; \mathrm{P}<.180(\mathrm{NS}), \mathrm{F}_{\text {groups }} \mathrm{x} \mathrm{F}_{\text {ages }} \mathrm{x} \mathrm{F}_{\text {sex }}: 2.048 ; \mathrm{P}<.158(\mathrm{NS})$

Between the groups a significant difference was observed in the mean Palpl-U1 values of DS and Normal individual where $\mathrm{DS}$ had lower value $(\mathrm{F}=38.455 ; \mathrm{P}<.000)$. Between age groups also a significant difference was observed $(\mathrm{F}=19.067 ; \mathrm{P}<.000)$ where 12-18 year group had higher value than 6-12 year. Between sex groups a non significant difference was observed. All the interaction effects were found to be non significant. 
Table 15: Mean Palpl-U6 values of male and female subjects belonging to different age group in Down's syndrome and Normal groups along with the results of $F$ test

\begin{tabular}{|c|c|c|c|c|c|c|c|}
\hline \multirow{2}{*}{$\begin{array}{l}\begin{array}{l}\text { Age } \\
\text { groups }\end{array} \\
6-12 \mathrm{yrs}\end{array}$} & \multirow[t]{2}{*}{ Sex } & \multicolumn{2}{|l|}{ DS } & \multicolumn{2}{|l|}{ Normal } & \multicolumn{2}{|l|}{ Overall } \\
\hline & & Mean & S.D & Mean & S.D & Mean & S.D \\
\hline & $\mathrm{M}$ & 15.2500 & 2.0529 & 17.8750 & 2.0310 & 16.5625 & 2.3936 \\
\hline & F & 17.6250 & 2.1339 & 17.0000 & 1.3093 & 17.3125 & 1.7405 \\
\hline & $\mathrm{T}$ & 16.4375 & 2.3656 & 17.4375 & 1.7115 & 16.9375 & 2.0936 \\
\hline \multirow[t]{3}{*}{$12-18 \mathrm{yrs}$} & M & 19.8750 & 1.1260 & 25.3750 & 3.7009 & 22.6250 & 3.8794 \\
\hline & $\mathrm{F}$ & 19.5000 & 4.2762 & 23.6250 & 3.3780 & 21.5625 & 4.2890 \\
\hline & $\mathrm{T}$ & 19.6875 & 3.0270 & 24.5000 & 3.5402 & 22.0937 & 4.0589 \\
\hline \multirow[t]{3}{*}{ Total } & $\mathrm{M}$ & 17.5625 & 2.8745 & 21.6250 & 4.8287 & 19.5938 & 4.4203 \\
\hline & $\mathrm{F}$ & 18.5625 & 3.4053 & 20.3125 & 4.2225 & 19.4375 & 3.8766 \\
\hline & $\mathrm{T}$ & 18.0625 & 3.1412 & 20.9687 & 4.5115 & 19.5156 & 4.1250 \\
\hline
\end{tabular}

\section{Tests of Between -Subjects Effects}

K) $\mathrm{F}_{\text {groups }}$ : 18.241; $\mathrm{P}<.000(\mathrm{~S}), \mathrm{F}_{\text {ages }}: 57.419 ; \mathrm{P}<.000(\mathrm{~S}), \mathrm{F}_{\text {sex }}: .053 ; \mathrm{P}<.816$ (NS)

$\mathrm{F}_{\text {groups }} \mathrm{x} \mathrm{F}_{\text {ages }}: 7.848 ; \mathrm{P}<.007(\mathrm{~S}), \mathrm{F}_{\text {groups }} \mathrm{x} \mathrm{F}_{\text {sex }}: 2.887 ; \mathrm{P}<.095(\mathrm{NS})$

$\mathrm{F}_{\text {ages }} \mathrm{x} \mathrm{F}_{\text {sex }}: 1.774 ; \mathrm{P}<.188(\mathrm{NS}), \mathrm{F}_{\text {groups }} \mathrm{x} \mathrm{F}_{\text {ages }} \mathrm{x} \mathrm{F}_{\text {sex }}: .475 ; \mathrm{P}<.494(\mathrm{NS})$

Between the groups a significant difference was observed in the mean Palpl-U6 values of DS and Normal individual where $\mathrm{DS}$ had higher value $(\mathrm{F}=18.241 ; \mathrm{P}<.000)$. Between age groups also a significant difference was observed $(\mathrm{F}=57.419 ; \mathrm{P}<.000)$ where 12-18 year group had higher value than 6-12 year. Between sex groups a non significant difference was observed $(\mathrm{F}=.053 ; \mathrm{P}<.816)$. There is a significant difference in the mean value of 12-18 year of DS and Normal as compared to 6-12 year of DS and Normal $(\mathrm{F}=4.434 ; \mathrm{P}<.040)$. In Normal group the values were higher in 12-18 year age than 6-12 year, whereas values were almost same in DS group. 
Graph 1: Mean NSBa values for Male and Female Subjects belonging to Down's syndrome and Normal group

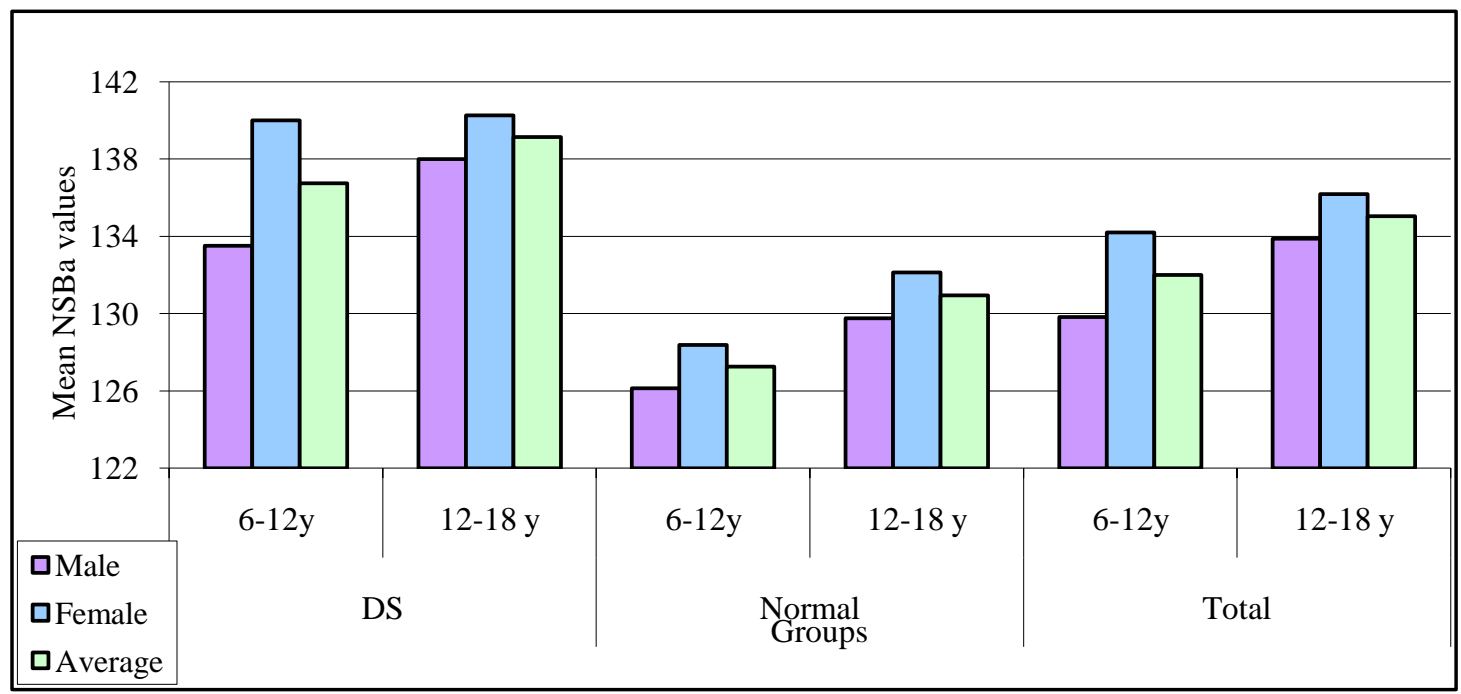

Graph 2: Mean SNA values for Male and Female Subjects belonging to Down's syndrome and Normal group

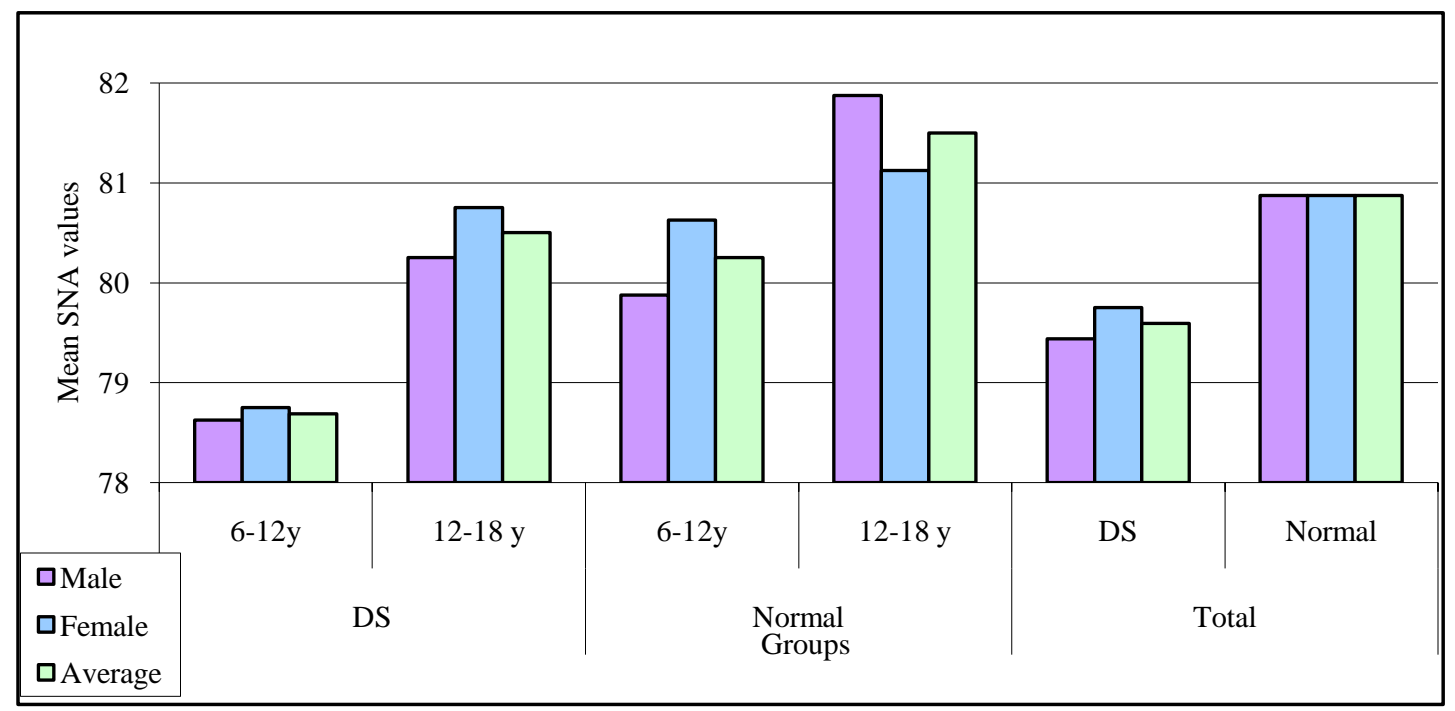

\section{DISCUSSION}

This cephalometric study on subjects with Down's syndrome was based on a sample of 64 subjects, 32 subjects of Down's syndrome and 32 Normal individuals in age group of $6-18$ years. None of them had previous history of any orthodontic treatment.

The age range for the sample size was divided into two groups 6-12 years and 12-18 years. 6-12 years represented the mixed dentition period and 12-18 years represented the permanent dentition. The two groups were analyzed to compare the growth changes in Down's syndrome subjects with that of normal. Strict criteria for inclusion were used in this study. 
Various cephalometric points, planes, angles and linear measurements were used in this study to evaluate the cranial base and maxilla to cranium relationships and dental characteristics of Down's syndrome subjects.

\section{Cranial base relationships}

A significant difference in the mean NSBa values of Down's syndrome and the normal group was found in the study. The study showed that there was a flatter cranial base in Down's syndrome group.

Results showed that the NSBa angle was on average more obtuse in Down's syndrome individuals when compared to that of normal. Hence presenting a flat cranial base in Down's syndrome group. Our results were in agreement with the results of Brandies, ${ }^{830}$ Burwood. ${ }^{925}$ Burwood showed that Down's syndrome group had disproportionate increase in the basal angle relative to their cranial capacity, which is in agreement with Alonso Tosso. ${ }^{10}$ According to Burwood Boogard's basal angle may therefore be used as a confirmatory sign in the radiological diagnosis of Down's syndrome. ${ }^{9}$

\section{Maxillary teeth to other structures}

The significant difference in the mean value of Palpl-U1 was observed between the Down's syndrome group and normal. The distance between the palatal plane and upper incisors was less in Down's syndrome group as compared to normal and the increase in this distance with age was found to be more in normal as compared to Down's syndrome. The reason for decreased eruption of upper incisors can be due to the macroglossia and tongue protrusion seen in Down's syndrome group, which results in relative intrusion of the upper incisors.

The eruption of upper molars was also found to be less in Down's syndrome group when compared to normal. The finding was in agreement with that of Spitzer. ${ }^{2}$

According to Brandies the Down's syndrome cranium was not found to be microcephalic at birth but showed lack of growth. The Down's syndrome child could not keep pace with the normal child and with age all Down's syndrome child appeared microcephalic. ${ }^{11}$

An early aging in Down's syndrome which was unique to the condition and appreciably in advance of the normal was suggested by Pozsonyi. ${ }^{12}$

Frosted found that the overall size of the craniofacial complex was smaller in Down's syndrome at 4 years of age and remained smaller into adulthood. Down's syndrome individual remain different throughout growth but did have growth at the same rate and in same direction as in normal group. ${ }^{1}$

\section{CONCLUSION}

From this cephalometric study of the comparison of cranial base, midface and dental characteristics between individuals with Down's syndrome and normal, we made the following conclusion:

1. There was significant difference found in the NSBa value between the Down's syndrome group and normal. The cranial base was significantly flatter in Down's syndrome group. 
2. The length of the anterior cranial base was found to be diminished in individuals with Down's syndrome when compared with normal. The growth of cranial base in Down's syndrome was less than that of normal.

3. The entire skull base length was found to be significantly decreased in individuals with Down's syndrome.

4. The SNA value in Down's syndrome was found to be similar to that of normal group. This showed that there was no difference in the position of the maxilla in both the groups.

5. The inclination of palatal plane was similar in Down's syndrome and normal and remained unchanged with age.

6. The upper anterior facial height was also found to be less in Down's syndrome group and the difference in upper anterior facial height between Down's syndrome and normal increased with age.

7. The length of the palate was significantly less in Down's syndrome group when compared with that of normal.

8. The upper incisors were more proclined and protruded in Down's syndrome group and the proclination of upper incisors increased with age in Down's syndrome group.

9. The distance between the upper incisors and palatal plane was found to be less in Down's syndrome group when compared to that of normal.

10. The upper molars were also found to be intruded in Down's syndrome group.

\section{REFERENCES}

1. Forstad W A, Cleal J F, Melosky L C. Craniofacial complex in the trisomy 21 syndrome. Archs. Oral. Biol 1971; 16: 707-22.

2. Spitzer R, Rabinowitch J Y, Wybar K C. A study of the abnormalities of the skull, teeth and lenses in mongolism. Canad M A J 1961; 84: 567-72.

3. Quintanilla J S, Biedma B M, Rofrigues M Q. Cephalometrics in children with Down's syndrome. PediatrRadiol 2002; 32: 635-43.

4. Jensen G M, Cleall J F, Yip A S. Dentoalveolar morphology and developmental changes in Down's syndrome (trisomy 21). Am J Orthod 1973; 64: 607-18.

5. Hutchinson, Cockburn. Practical pediatric problems. $6^{\text {th }}$ ed. P G Publications. 1986.

6. Fink G B, Madaus W K, Walker G F. A quantitative study of the face in Down's syndrome. Am. J. Orthod 1975; 67: 540-43.

7. Kaplan, Sadock. Comprehensive textbook of psychiatry. $6^{\text {th }}$ ed. Williams \& Wilkins.

8. Brandies H F, Schmid R G, Brandies E F. Craniofacial development in patients with Down's syndrome from birth to 14years of age. Eurp J Orthod 1986; 8: 35-42.

9. Burwood R J, Gordon I R S, Taft R D. The skull in mongolism. ClinRadiol 1973; 24: 475-80.

10. Tosso A A, Gias N L, Vallejo H G, Tomas L M. Cephalometric study of the cranial base in 133 cases of Down's Syndrome. Rev StomatolChirMaxillofac. 1985; 86: 234-40

11. Brandies H F. Cephalometric comparison between children with and without Down's syndrome. Eur J Orthod 1988; 10: 255-63.

12. Pozsonyi J, Gibson D, Zarfas D E. Skeletal maturation in mongolism (Down's syndrome). J Pediat 1964; 64: 75-78. 